\title{
Schedule-induced and metabolic polydipsia
}

\author{
MICHAEL E. BRUSH and ROBERT W. SCHAEFFER \\ Auburn University, Auburn, Alabama 36830
}

\begin{abstract}
Two female albino rats, one (Rat FF) allowed free access to food throughout the experiment and the other (Rat FD) maintained at $80 \%$ of its free-feeding weight, were exposed to (1) baseline conditions with water freely available in both the home cage and test chamber and (2) baseline and free-reinforcement schedule (FFI-60-sec) conditions with free access to water in the test chamber and no access to water in the home cage. While home-cage water deprivation produced metabolic polydipsia in both subjects during test-chamber sessions, free-reinforcement schedule conditions produced schedule-induced polydipsia in Rat FD only. When under home-cage water-deprivation conditions (2), test-chamber water intakes of the subjects differed only during the free-reinforcement schedule condition even though both subjects had equal food-ingestion rates in the test chamber. Results were discussed with respect to previous explanations of schedule-induced polydipsia as a form of metabolic polydipsia.
\end{abstract}

Water intakes can be accelerated in animals by concurrently placing them under a regimen of food deprivation and exposing them to certain schedules of intermittent food reinforcement (schedule-induced polydipsia; Falk, 1961). Similarly, animals deprived of water for extended periods of time demonstrate accelerated intakes when finally allowed access to water (metabolic polydipsia; Stellar \& Hill, 1952). Although the determinants of metabolic polydipsia are clearly physiological (e.g., cellular fluid imbalance, oropharyngeal dryness), the exact role of such determinants in schedule-induced polydipsia remains unresolved. Although the development and maintenance of schedule-induced polydipsia have previously been attributed to the synergistic effect of spaced feeding and the animal's natural tendency to drink after eating (Lotter, Woods, \& Vasselli, 1973; Stein, 1964) and salivary insufficiency and digestive inefficiency (Teitlebaum, 1966), Brush and Schaeffer (1974) have contended that, while such metabolic determinants may increase the probability of the development of scheduled-induced polydipsia, they are not sufficient to account for the phenomenon. The present experiment was designed to further investigate the differences between schedule-induced and metabolic polydipsia.

\section{METHOD}

\section{Subjects}

Two, experimentally naive, Sprague-Dawley, female albino rats, approximately 150 days old at the onset of the experiment, were used. Rat FF was allowed free access to food in the home cage throughout the experiment; Rat FD was maintained at $80 \%$ of its free-feeding weight throughout the experiment.

\section{Apparatus \\ A standard operant conditioning chamber, equipped with an inoperative lever, a food magazine, and an automatic feeder, was used. A water bottle was at tached to the outside of the chamber by a plastic positioning collar with the spout of the drinking}

Requests for reprints should be sent to Robert W. Schaeffer. tube slightly recessed within a $1.27-\mathrm{cm}$ diam opening in the chamber wall and located $4 \mathrm{~cm}$ to the right of the food magazine and $5 \mathrm{~cm}$ from the floor of the chamber. Delivery of Noyes 45-mg "sugarless" pellets was automatically programmed by standard relay circuitry; licks and pellet deliveries were recorded on counters and cumulative recorders.

\section{Procedure}

The experiment consisted of five phases: (1) Baseline 1, (2) Baseline 2, (3) FFI-60-sec phase, (4) reinstatement of Baseline 2, and (5) reinstatement of Baseline 1. All test-chamber sessions were 100 minutes in duration. Water was always freely available to the subjects in the test chamber.

During Baseline 1 and Baseline 2, 100 food pellets were placed in the food magazine, and the empty feeder was operated on a free-fixed-interval schedule (FFI-60-sec). Water was freely avaiable to both subjects in their home cages during Baseline 1; during Baseline 2, the water bottles were removed from both subjects' home cages, placing them on $22.33 \mathrm{~h}$ period of water deprivation prior to each test chamber session.

During the FFI-60-sec phase, the home-cage water conditions used in Baseline 2 remained in effect, while test-chamber sessions consisted of 100 food pellets delivered individually and noncontingently at 60 -sec intervals to each subject (FFI-60-sec schedule).

During the reinstatements of Baseline 2 and Baseline 1, the test-chamber and home-cage conditions previously employed in Baseline 2 and Baseline 1 were respectively replicated.

Test-chamber water intakes were measured immediately after each session by weight differences in the water bottle and converted into milliliters. Observations of the pattern and quantity of food consumption in the test chamber were made both during and after each session. Each phase of the experiment remained in effect until both subjects' test-chamber water intakes had stablized. Stability was defined as the difference between the total intake of the last three sessions and previous three sessions of each phase being less than $20 \%$ of the total intake of the last six sessions.

\section{RESULTS AND DISCUSSION}

Both subjects' test-chamber water intakes during each phase of the experiment are presented in Figure 1. During Baseline 1, Rat FD ingested more water than Rat FF. The subjects' patterns and quantities of food consumption also differed. Rat FD consumed all available food pellets during the initial portion of each 


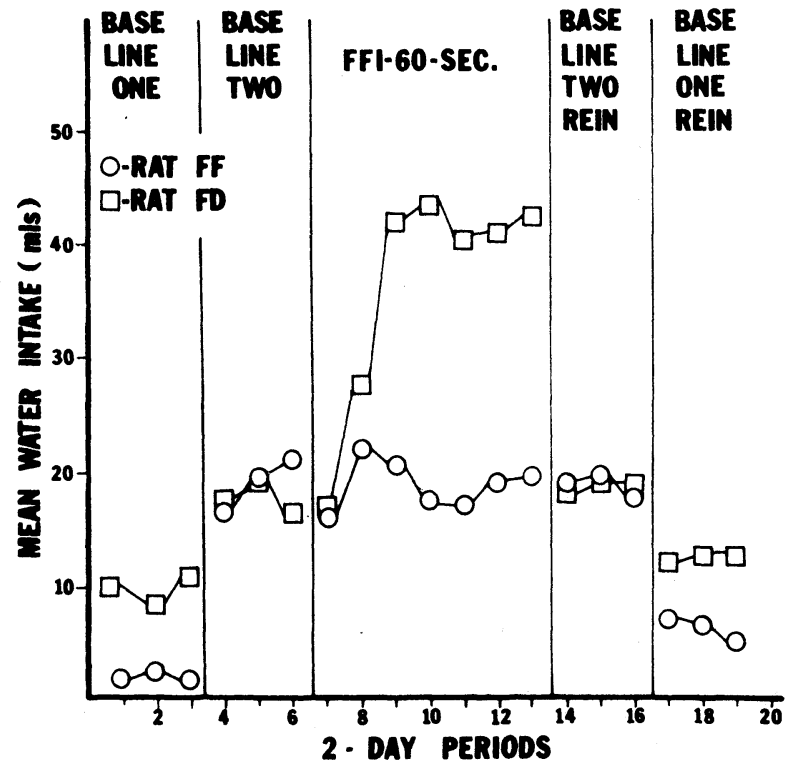
FD.

Figure 1. Mean test-chamber water intakes for Rat FF and Rat

chamber session; in contrast, Rat FF tended to sporadically consume food pellets throughout the session, never consuming more than half of the available food pellets during any session.

During Baseline 2, the introduction of home-cage water deprivation produced immediate increments in both subjects' test-chamber water intakes, relative to Baseline 1, and essentially eliminated the difference between the two subjects' levels of test-chamber water intake that was observed in Baseline 1. A concurrent change in food consumption was also observed in Rat FF; during Baseline 2, all food pellets presented to each subject during each session were consumed. These alterations in food consumption and water intake illustrate the strong temporal and quantitative relationships between food and water ingestion that have previously been reported (e.g., Fitzsimons \& LeMagnen, 1969).

Although home-cage water deprivation during Baseline 2 produced increased test-chamber water intakes (i.e., metabolic polydipsia) in both subjects, relative to Baseline 1, the combined home-cage water-deprivation and intermittent reinforcement schedule conditions of the FFI-60-sec phase produced further increases in water intakes in Rat FD only (i.e., schedule-induced polydipsia), even though both subjects continued to consume all available food pellets. The dramatic difference in test-chamber water intakes between the two subjects during the FFI-60-sec phase strongly argues against any explanation of schedule-induced polydipsia as a form of metabolic polydipsia. If schedule-induced polydipsia results from the synergistic effect of spaced feeding and thirst (Stein, 1964), there should have been no major difference between the subjects' water intakes since both subjects consumed identical quantities of food. If schedule-induced polydipsia results from salivary insufficiency and digestive inefficiency (Teitlebaum, 1966), there should have been no difference between the subjects' water intakes since (a) both subjects' access to water in the home cage had been eliminated, (b) both subjects' digestive and salivary mechanisms, therefore, were hindered, and (c) both subjects consumed identical quantities of food.

During the reinstatement of Baseline 2, the removal of the intermittent-reinforcement-schedule condition produced a decrease in Rat FD's water intake (relative to the preceeding phase) to a level commensurate with that of the original Baseline 2. Rat FF's level of water intake remained essentially unaltered, relative to the two preceeding phases. Reinstatement of Baseline 1 conditions resulted in decreases in both subjects' water intakes with the difference between the two subjects' water intakes being equivalent to the intake difference evident during the original Baseline 1 condition. Both subjects continued to consume all food food pellets available in the test chamber during the reinstatements of both Baseline 1 and Baseline 2.

Statistical analyses (i.e., ANOVA, Duncan's Multiple Range Test) indicated that the mean phase intakes of the two subjects were significantly different $(p<.01)$ during only Baseline 1, its reinstatement, and the FFI-60-sec phase. For Rat FF, there were significant differences $(p<.01)$ between the mean phase intakes of Baseline 1 and (1) Baseline 2 and (2) the FFI-60-sec phase, but no significant difference $(\mathrm{p}>.05)$ between the mean phase intakes of Baseline 2 and FFI-60-sec phase. For Rat FD there were significant differences $(p<.01)$ between the mean phase intakes of all of the first three phases of the experiment. Since (a) the significant difference between the two subjects' mean phase intakes during the FFI-60-sec phase indicates that food deprivation (the only experimental difference between the subjects) is a necessary condition for schedule-induced polydipsia and (b) intermittent reinforcement schedules are aversive to food-deprived animals (Appel, 1963), it seems evident that schedule-induced polydipsia is a motivational, rather than physiological, phenomenon.

\section{REFERENCES}

Appel, J. B. Aversive aspects of a schedule of positive reinforcement. Journal of Experimental Analysis of Behavior, 1963, 6, 423-428

Brush, M. E., \& Schaeffer, R. W. Effects of water deprivation on schedule-induced polydipsia. Bulletin of the Psychonomic Society, 1974, 4, 69-72.

Falk, J. L. Production of polydipsia in normal rats by an intermittent food schedule. Science. 1961. 133. 195-196.

Fitzsimons, T. J., \& LeMagnen, J. Eating as a regulatory control of drinking in the rat. Journal of Comparative and Physiological Psychology, 1969, 67, 273-283.

Lotter, E. C., Woods, S. C., \& Vasselli, J. R. Schedule-induced polydipsia: An artifact. Journal of Comparative and Physiological Psychology, 1973, 83, 478-484.

Stein, L. Excessive drinking in the rat: Superstition or thirst? 
Journal of Comparative and Physiological Psychology, 1964, $58,237-242$.

Stellar, E., \& Hill, J. H. The rat's rate of drinking as a function of water deprivation. Journal of Comparative and Physiological Psychology, 1952, 45, 96-102.

Teitlebaum, P. The use of operant methods in the assessment and control of motivational states. In W. K. Honig (Ed.), Operant behavior: Areas of research and application. New York: A pplet on-Century-Crofts, 1966.

(Received for publication October $31,1974$. )

\title{
Semantically cued retrieval of words from long-term memory
}

\author{
D. J. MURRAY \\ Queen's University, Kingston, Ontario, Canada
}

\begin{abstract}
Subjects were asked to give in 2 min as many words as possible which belonged to a given category such as "girls' names." It was found that the number of words reported was a power function of the number of words estimated to be in the category, and that high frequency words were given before low frequency words.
\end{abstract}

It has been found by Standing (1973) that the number of items which can be retrieved from memory is a power function of the number of items presented. If $\mathrm{R}$ is the number retrieved and $P$ is the number presented,

$$
\mathrm{R}=\mathrm{kPm}
$$

where $\mathrm{m}$ varies with the experimental paradigm. Murray (1975) also examined retrieval by a "free output" method: subjects were asked to find as many words as they could, in $2 \mathrm{~min}$, whose $1 \mathrm{st}, 2 \mathrm{nd}, 3 \mathrm{rd}$, 4 th, or 5 th letter was A or B or ... or Z. For all five levels of retrieval cue, the number of words emitted was a power function of the number of words having the required letter properties presumed to be known by the subject.

The purpose of the present paper is to examine the free output of words given semantic rather than graphemic retrieval cues. Although Loftus, Wiksten, and Abelson (1974) have shown that there may be differences in search techniques when one is attempting to retrieve a word cued graphemically as opposed to

This research was supported by National Research Council of Canada Grant A0126. The paper is sponsored by P. C. Dodwell, who takes full editorial responsibility for it. The author is most grateful for resarch assistance to W. E. Hockley and S. Moore. Reprint requests should be sent to D. J. Murray, Psychology Department, Queen's University, Kingston, Ontario, Canada K7L 3N6. semantically, we need to discover experimentally if free output cued semantically follows a power function in the same way as does free output cued graphemically. With graphemic cues, the index of vocabulary size (the number of words with the appropriate characteristics presumably known to the subject) was given by a count of the words in the Thorndike Lorge lists. With semantic cues, such a count, for example, of all the names of trees or animals, would be tedious and probably inaccurate. Instead, the category norms of Battig and Montague (1969) were used to estimate the probable numbers of words of a given category likely to be known to student subjects. These norms also give the relative frequencies with which each category member comes to mind given a category name, so than an examination of frequency effects over time can also be undertaken.

\section{METHOD}

\section{Materials}

Of the 56 categories in the Battig and Montague norms, 30 were selected on the basis of their giving a wide range of vocabulary sizes. In the norms, the words in each category are divided into two sections, those words produced by 10 or more subjects in the original population, and those words produced by fewer than 10 subjects. In the present study, the former subset of words constituted the value of "vocabulary size." For example, there were 18 words given by more than 10 subjects in answer to the category "a precious stone" although a total of 68 words was given in all, including many words given by only one 